\title{
PRESENTATIONS OF $n$-KNOTS
}

\author{
BY \\ C. KEARTON
}

\begin{abstract}
The method of critical level embeddings is used to generalize the technique of knot presentations from the classical case to the case of $n$-knots. For $n>3$, it is shown that an $n$-knot with algebraically simple complement has a correspondingly simple presentation.
\end{abstract}

0. Introduction. There are two main approaches to classical knot theory: the method of presentations and the use of the Seifert surface. The latter has. been employed by several authors (cf. Kervaire [7], Levine [8], [9], [10]) to study knots of higher dimensions, i.e., $n$-knots.

Working in the piecewise linear category throughout, we shall develop a theory of presentations for $n$-knots. If we consider an $n$-knot as a locally flat embedding of $S^{n}$ in $S^{n+1} \times I$, then a presentation of the knot is a critical level embedding; i.e., one which, regarding $S^{n}$ as $S^{n}=$ handle + collar + handle + collar $+\cdots$, embeds each handle in a level of $S^{n+1} \dot{x} I$ and each collar productwise along the $I$ direction.

For the case $n=1$, we obtain what is essentially a classical presentation: the main difference is that we allow vertical collars. For $n=2$, we obtain the description of 2-knots given by Fox [2].

We call an $n$-knot $r$-simple if its complement has the homotopy $r$-type of a circle, and we call a presentation $r$-simple if it has only one handle of $S^{n}$ of index less than $r$ (there must always be at lease one 0 -handle).

Generalizing the techniques of Fox for 2-knots [2], we may use a presentation of an $n$-knot to obtain presentations of its group and of the homology modules associated with the infinite cyclic cover of the complement. By this means it is not hard to see that a knot with an $r$-simple presentation is $r$-simple. Conversely, Theorem 5.1 shows that any $r$-simple $n$-knot, $n>3,2 r<n$, has an $r$-simple presentation: this is proved by eliminating handles of $S^{n}$ in order of increasing index from a given presentation, as in the proof of the s-cobordism theorem. At each stage there is an obstruction measured by elements in a group ring.

Received by the editors January 11, 1974.

AMS (MOS) subject classifications (1970). Primary 57C45; Secondary 55^05, 55A25. Key words and phrases. $n$-knot, knot presentation. 
This theorem has a parallel in a result of Levine [8], who shows that the $S^{n}$ above bounds an $r$-connected locally flat submanifold of $S^{n+2}$.

The dual of a presentation is obtained by reversing the orientations of $S^{n+1}$ and $I$ in $S^{n+1} \times I$; i.e., by turning the given presentation upside down. This is a generalization of the dual presentation of Fox and Torres [12] in the classical case. Applying the theorem above to the dual presentation, we see that the knot has a presentation with only handles of index $i, r \leqslant i \leqslant n-r$, in the middle dimensions.

In a subsequent paper, we shall apply the techniques and results of this paper to obtain a classification of $(q-1)$-simple $(2 q-1)$-knots for $q>3$; these are the simple knots of Levine [10]. These results, together with those of the present paper, were announced in [5].

1. Preliminaries. We shall work throughout in the piecewise linear category, and we shall assume that all submanifolds are locally flat. Let $S^{r}$ denote an $r$ sphere. An $n$-knot is an oriented pair $\left(S^{n+2}, S^{n}\right)$, where $S^{n}$ is a submanifold of $S^{n+2}$. Two knots are equivalent if there is an isomorphism of pairs between them which preserves orientations.

By removing the interiors of two disjoint $(n+2)$-balls from the complement of a regular neighborhood of $S^{n}$, we obtain $S^{n} \subset \operatorname{int}\left(S^{n+1} \times n\right)$, where $I$ denotes the unit interval $[0,1]$. A presentation of the $n$-knot is a critical level embedding. This is an embedding which, regarding $S^{n}$ as $S^{n}=$ handle + collar + handle + collar $+\cdots$, embeds each handle in a level of $S^{n+1} \times I$ and each collar productwise along the $I$ direction. Further details may be found in the papers of Rourke [11] or Kearton and Lickorish [6].

Henceforth, $K$ will denote the complement in $S^{n+2}$ of the interior of a regular neighborhood of $S^{n}$. By Alexander-Poincaré duality, $H_{1}(K)$ is the infinite cyclic group, which we write multiplicatively as ( $t$ : ). Having chosen orientations for $S^{n}$ and $S^{n+2}$, we are provided with a natural choice for the generator of $H_{1}(K)$, by the duality theorems, and we choose $t$ to be this generator.

$\widetilde{K}$ will denote the cover of $K$ corresponding to the kernel of the Hurewicz map $\pi_{1}(K) \rightarrow H_{1}(K)$, so that $\widetilde{K}$ is an infinite cyclic cover of $K$.

We shall always use the integers as coefficients for homology, and we shall denote the integral group ring of the group $(t:)$ by $\mathbf{Z}[t]$.

The group presentation with generators $x_{1}, \cdots, x_{r}$ and relations $r_{1}, \cdots$, $r_{s}$ will be written as $\left(x_{1}, \cdots, x_{r}: r_{1}, \cdots, r_{s}\right)$ and the $\mathrm{Z}[t]$-module presentation as $\left[x_{1}, \cdots, x_{r}: r_{1}, \cdots, r_{s}\right]$.

2. Critical levels. Let $Q$ be a closed ( $q-1)$-manifold, and $M$ a compact proper submanifold of $Q \times I$, of dimension $m<q$. Assume further that 
$M$ is in critical level position with just one critical level, of index $r$, at level $1 / 2$. Thus we have

$$
M_{0} \times[0,1 / 2] \subset Q \times[0,1 / 2], \quad M_{1} \times[1 / 2,1] \subset Q \times[1 / 2,1],
$$

the inclusion maps having the form (inclusion $\times$ identity), and an $r$-handle $B^{r} \times$ $B^{m-r}$ of $M$ with $B^{r} \times B^{m-r} \subset Q \times 1 / 2$.

We take the opportunity to introduce some notation.

$A=$ int $A=$ interior of $A$.

$\bar{A}=\mathrm{cl} A=$ closure of $A$.

$N(R ; T)=$ regular neighborhood of $R$ in $T$.

$N(R, S ; T)=$ regular neighborhood of $R \bmod S$ in $T$.

$H_{(t)}=H \cap(Q \times[0, t])$.

$H_{t}=H \cap(Q \times t)$.

$\beta B^{s}=[-\beta, \beta]^{s}$ for $\beta>0$.

$H_{t} \times r=$ projection of $H_{t}$ into $Q \times r=$ shadow of $H_{t}$ in level $r$.

We remark that in the situation above we may assume that $N(M ; Q \times I)$ is the union of the following spaces.

$$
N\left(M_{0} ; Q_{0}\right) \times[0,1 / 4], \quad N\left(M_{1 / 2} ; Q_{1 / 2}\right) \times[1 / 4,3 / 4], \quad N\left(M_{1} ; Q_{1}\right) \times[3 / 4,1] .
$$

Henceforth when dealing with critical level embeddings, and in particular with presentations of $n$-knots, we shall always make this assumption when considering regular neighborhoods.

LEMMA 2.1. In the situation above, $\operatorname{cl}(Q \times I-N(M ; Q \times I))$ is isomorphic

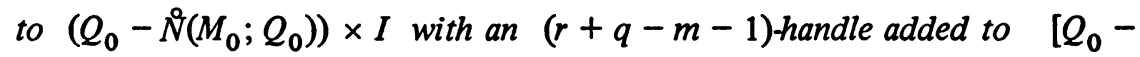
$\left.N\left(M_{0} ; Q_{0}\right)\right] \times 1$.

Proof. Suppose that we triangulate $Q \times I$ so that it collapses simplicially to $Q \times 1$, with $M$ triangulated as a subcomplex. Each elementary collapse of a simplex $\alpha$ from one of its faces $\beta$ falls into one of three categories: both $\alpha$ and $\beta$ are in $M$, neither are in $M$, or $\beta$ is in $M$ but $\alpha$ is not. We may arrange for the third case to occur just once, with an $(m-r)$-simplex. The work of Kearton and Lickorish [6] shows that $Q \times I$ has a handle decomposition on $Q \times 1$ with just two handles: an $(m-r)$-handle which is a regular neighborhood of the barycentre of $\beta$, and an $(m-r+1)$-handle which does not meet $M$. Dualizing to the handle decomposition on $Q \times 0$, we obtain the desired result.

Suppose that we are given an ambient isotopy of $Q \times 1 / 2$ which keeps $M_{0} \times 1 / 2$ setwise invariant. We may extend this to an ambient isotopy of $Q \times$ $[1 / 2,1]$ by (isotopy $\times$ identity). By a collaring argument, we may extend to $Q \times[0,1 / 2]$ keeping $Q \times 0$ fixed and $M_{0} \times[0,1 / 2]$ setwise invariant. We thus 
keep $M$ in critical level position, except for the fact that $M_{0} \times[0,1 / 2]$ may no longer be embedded by (inclusion $x$ identity). But we shall be concerned with the submanifold rather than the embedding, and so this may be dealt with by reparametrizing the collar $M_{0} \times[0,1 / 2]$.

LEMMA 2.2. Given an ambient isotopy of $\partial B^{r} \times B^{m-r}$ in $M_{0} \times 1 / 2$, we may extend this to an ambient isotopy of $B^{r} \times B^{m-r}$ in $Q \times I$, keeping $Q \times$ 0 fixed.

Proof. First recall that an ambient isotopy may be realized as an isotopy by moves [3, Theorem 6.2]. If $B^{m-1} \subset M_{0} \times 1 / 2$ is the support of a move, extend this move to

$$
N\left(B^{m-1}, \partial B^{m-1} ; Q \times 1 / 2\right) \cong B^{q-m} \times B^{m-1}
$$

by (identity $\times$ move). Extend to $N\left(M_{0} \times 1 / 2 ; Q \times 1 / 2\right)$ by the identity (the move is fixed on $\left.\partial B^{m-1}\right)$, and use a collar of $N\left(M_{0} \times 1 / 2 ; Q \times 1 / 2\right)$ to extend this isotopy to $Q \times 1 / 2$. The remarks above apply to complete the proof.

By a result of Rourke [11], we may isotop a critical level embedding of a manifold $M$ in $Q \times I$ until the handles are added in order of increasing index, handles of the same index being added at the same level, provided $q-m>1$. In particular, we may do this for $n$-knots, and henceforth we shall consider only presentations which have this form.

REMARK 2.3. Suppose that $M$ is embedded in $Q \times I$, in critical level position, and that two handles which in the abstract handle decomposition of $M$ form a cancelling pair may be isotoped into the same level of $Q \times I$. Then by a result of Rourke [11] we may cancel this pair in the embedded situation, leaving $M$ in critical level position. An alternative proof may be given via the collapsing procedure of [6].

Recall that $I=[0,1]$, so that we have standard inclusions

$$
I \subset B^{1}=B^{1} \times 0 \subset B^{1} \times B^{1}=B^{2} \text {. }
$$

Similarly for a manifold $M$ we have standard inclusions

$$
I \times 0 \times M \subset B^{1} \times 0 \times M C B^{2} \times M,
$$

each map being (inclusion $x$ identity).

We now consider a presentation of an $n$-knot. Recall that an $n$-knot has trivial normal bundle, and assume that after all the handles of index at most $(r-1)$ have been added, we are left with a manifold $M \subset S^{n+1}$ for some level $S^{n+1}$, where $M$ has a trivial normal bundle inherited from that of $S^{n}$.

Let $B^{r} \times B^{n-r} \subset S^{n+1}$ be an $r$-handle of the knot. By taking secondderived neighborhoods in some triangulation, we may assume that 


$$
\left(B^{r} \times B^{n-r}\right) \cap\left(B^{2} \times M\right)=I \times \partial B^{r} \times B^{n-r},
$$

where $B^{2} \times M$ is the normal bundle of $M$ and $I \times B^{r} \times B^{n-r}$ is a regular neighborhood of $\partial B^{r} \times B^{n-r}, \bmod \partial B^{r} \times \partial B^{n-r}$, in $B^{r} \times B^{n-r}$.

We shall say that the $r$-handle (or its core) is in normal position if, in addition,

$$
\begin{aligned}
& I \times \partial B^{r} \times B^{n-r} \subset I \times 0 \times M \subset B^{2} \times M \\
& \quad\left(\text { or } I \times \partial B^{r} \times 0 \subset I \times 0 \times M \subset B^{2} \times M\right)
\end{aligned}
$$

by (identity $x$ inclusion) and (inclusion $x$ identity).

We wish to show that in the circumstances outlined above, the $r$-handle may always be moved into normal position. By the results of [4], we may assume that the presentation has come about by means of a collapse of $S^{n+1} \times I$ to $S^{n+1} \times$ 0 . If $a * \Delta$ is the $(r+1)$-simplex which gives rise to the $r$-handle, so that $\Delta$ is an $r$-simplex of $S^{n+1}$ and $a$ is a vertex not in $S^{n}$, we may arrange for $a$ to lie in $1 \times 0 \times M \subset B^{2} \times M$. Following the proof of [4, Lemma 1], we may arrange for $I \times B^{r} \times B^{n-r} \subset I \times B^{1} \times M$, and finally we apply the theorems on compatible collars [3] to achieve the desired result.

We shall assume henceforth that, where appropriate, handles are in normal position.

3. Presentation of the knot group. Consider a presentation of an $n$-knot, $n \geqslant 3$, and let $\sigma$ be a level between the 0 and 1-handles of the knot (i.e., of $S^{n}$ ) and $\tau$ a level between the 1 and 2-handles.

LEMMA 4.1. $i_{*}: \pi_{1}\left(K_{\tau}\right) \rightarrow \pi_{1}(K)$ is an isomorphism.

Proof. By Lemma 2.1, $K_{(\tau)}$ is obtained from $K_{\tau} \times I$ by adding $n$ and $(n+1)$-handles, corresponding to the duals of the 0 and 1-handles of the knot. Thus $i_{*}: \pi_{1}\left(K_{\tau}\right) \rightarrow \pi_{1}\left(K_{(\tau)}\right)$ is an isomorphism. Similarly, $K_{(1)}$ is obtained from $K_{(\tau)}$ by adding handles of index greater than 2 , and so $i_{*}: \pi_{1}\left(K_{(\tau)}\right) \rightarrow$ $\pi_{1}\left(K_{(1)}\right)$ is an isomorphism. $K$ is obtained by adding two $(n+2)$-handles to $K_{(1)}$, and so $i_{*}: \pi_{1}\left(K_{(1)}\right) \rightarrow \pi_{1}(K)$ is an isomorphism.

Let $h_{i}^{q}$ denote the $i$ th $q$-handle of the knot. By the shadow of $h_{i}^{1}$ we shall mean its shadow in the level of the 0 -handles. By general position, we may assume that the shadows of the cores of the 1 -handles meet the interiors of the 0 -handles transversely in interior points and nowhere else.

We may compute $\pi_{1}\left(K_{\tau}\right)$ in the following way. Let $\rho$ be the level at which the 1-handles are added, and let $X$ be the closed complement in level $\rho$ of $N\left(S_{\rho}^{n} ; S^{n+1} \times \rho\right)$. Suppose that there are $p$ 0-handles and $m 1$-handles. We denote the $i$ th 1 -handle by $\left(B^{1} \times B^{n-1}\right)_{i}$, and it has a regular neighborhood in level $\rho$ of the form $B^{1} \times(1+\epsilon)\left(B^{1} \times B^{n-1}\right)_{i}$, where $\epsilon$ is small and 
positive. The intersection of $S^{n}$ with the level $\sigma$ is a set of $(n-1)$-spheres $S_{j}^{n-1}, 1 \leqslant j \leqslant p$, unknotted and unlinked. We may assume that the shadow of $B^{1} \times(1+\epsilon)\left(B^{1} \times B^{n-1}\right)_{i}$ meets a regular neighborhood of these spheres in the shadow of

$$
B^{1} \times\left(\mathrm{cl}\left[(1+\epsilon) B^{1}-(1-\epsilon) B^{1}\right] \times B^{n-1}\right){ }_{i} .
$$

We have the following homotopy equivalences, proved by deformation retracting $B^{1} \times(1+\epsilon)\left(B^{1} \times B^{n-1}\right)_{i}$ onto appropriate subspaces.

$$
\begin{aligned}
& K_{\sigma} \simeq X \cup \bigcup_{i=1}^{m} B^{1} \times(1+\epsilon)\left(0 \times B^{n-1}\right)_{i}, \\
& K_{\tau} \simeq X \cup \bigcup_{i=1}^{m} B^{1} \times(1+\epsilon)\left(B^{1} \times 0\right)_{i} .
\end{aligned}
$$

Now $\pi_{1}\left(K_{\sigma}\right)=\left(x_{1}, \cdots, x_{p}:\right)$, the free group on $p$ generators. Applying Van Kampen's theorem, we see that $\pi_{1}(X)=\pi_{1}\left(K_{\sigma}\right)$, as $n \geqslant 3$. Another application of the theorem shows that $\pi_{1}\left(K_{\tau}\right)=\left(x_{1}, \cdots, x_{p}: r_{1}, \cdots, r_{m}\right)$, where the relation $r_{j}$ is given by the 2-cell $B^{1} \times(1+\epsilon)\left(B^{1} \times 0\right)_{j}$.

Recalling Lemma 4.1 above, we define $\varphi$ to be the composite of the quotient and Hurewicz maps

$$
\varphi:\left(x_{1}, \cdots, x_{p} \overline{:}\right) \rightarrow\left(x_{1}, \cdots, x_{p}: r_{1}, \cdots, r_{m}\right) \rightarrow(t:) .
$$

Choose a point $a \in$ int $K_{\sigma}$ whose shadow does not lie in any 0 -handle; for each $i$, choose a little loop in $K_{\sigma}$ which links $S_{i}^{n-1}$ once in the positive direction, and whose shadow meets the $j$ th 0 -handle in a single point if $i=j$, but misses it otherwise. Choose a path in $K_{\sigma}$ from $a$ to the $i$ th loop, whose shadow misses all the 0 -handles; then the $i$ th path and loop represent an element $x_{i}$ of $\pi_{1}\left(K_{\sigma}, a\right)$, and the elements $x_{1} ; \cdots, x_{p}$ form a basis for this group. We write $\pi_{1}\left(K_{\sigma}\right)$ for $\pi_{1}\left(K_{\sigma}, a\right)$.

Note that $\varphi\left(x_{i}\right)=t$ for all $i$.

Suppose that $h_{j}^{1}$ joins $h_{i}^{0}$ and $h_{k}^{0}$ : orient the core of $h_{j}^{1}$ so that traversing the core in the positive direction means we pass from $h_{i}^{0}$ to $h_{k}^{0}$. Then the intersections that the shadow of the core of $h_{j}^{1}$ makes with the interiors of the 0 -handles defines a word $w$ in $x_{1}, \cdots, x_{p}$. Let $l_{j}$ denote the shadow in level $\sigma$ of $\partial\left(B^{1} \times(1+\epsilon)\left(B^{1} \times 0\right)_{j}\right)$, the boundary of the 2-cell mentioned above. If we are careful in choosing an orientation for $l_{j}$, and a point $b_{j}$ from which to start, then the intersections which the shadow of $l_{j}$ makes with the 0-handles defines the word $w x_{k} w^{-1} x_{i}^{-1}$. Choose a path from $a$ to $b_{j}$ whose shadow misses the 0-handles. Then this path and $l_{j}$ define the element $w x_{k} w^{-1} x_{i}^{-1}$ of $\pi_{1}\left(K_{\sigma}\right)$. This is the relation $r_{j}$, which we also write as $x_{i}=w x_{k} w^{-1}$. Call $w$ the conjugate part of the relation. 
As words in $x_{1}, \cdots, x_{p}$, these relations describe the intersections that the shadows of the cores of the 1-handles make with the 0-handles. As elements of $\pi_{1}\left(K_{\sigma}\right)$, we could reduce these words, i.e. replace $u x x^{-1} v$ by $u v$. We shall keep the relations as they are obtained from the intersections, but we shall show that by means of isotopy those intersections may be altered so as to effect a reduction. In these circumstances we shall say that reduction is permitted.

Lemma 3.2. Reduction is permitted in the conjugate parts of relations.

Proof. Suppose that $r_{j}$ has the form $x_{i}=w x_{k} w^{-1}$, and that $w=$ $u x_{h} x_{h}^{-1} v$. We have to show that $h_{j}^{1}$ may be isotoped to give the relation $x_{i}=$ $u v x_{k} v^{-1} u^{-1}$. The 1-dimensional core of $h_{j}^{1}$ cannot link with itself, or with the core of any other 1-handle, as $n \geqslant 3$, so there is no obstruction to isotoping $h_{j}^{1}$ so that the relevant intersections are described by $u v$. Moreover, none of the other relations are affected.

LEMmA 3.3. If $r_{j}$ has the form $x_{i}=w x_{k} w^{-1}$, and $w=u x_{k}$, then $h_{j}^{1}$ may be isotoped to yield $x_{i}=u x_{k} u^{-1}$. A similar result is true for $w=u x_{k}^{-1}$.

Proof. Consider how $h_{j}^{1}$ meets the unknotted sphere $S^{n-1}$ whose shadow is $S_{k}^{n-1}$. If $B^{n-1}=h_{j}^{1} \cap S^{n-1}$, a regular neighborhood of $B^{n-1} \bmod$ $\partial B^{n-1}$ in its level is of the form $B^{2} \times B^{n-1}$. Let $g_{t}: B^{2} \rightarrow B^{2}$ be an isotopy which rotates $\partial B^{2}$ once around itself and keeps the origin fixed. Then we apply $\left(g_{t} \times\right.$ identity) to $B^{2} \times B^{n-1}$, using collars to extend to the rest of the regular neighborhood of $S^{n-1}$, and then to the rest of the level. This has the effect of rotating the end of the core of $h_{j}^{1}$ once around $S^{n-1}$, to give the relation $x_{i}=u x_{k} u^{-1}$, using Lemma 3.2.

LEMMA 3.4. If $r_{j}$ has the form $x_{i}=x_{k}, i \neq k$, then we may cancel $h_{i}^{0}$ and $h_{j}^{1}$.

Proof. There is no obstruction to sliding $h_{j}^{1}$ down to the level of the 0-handles, and so cancelling, by Remark 2.3.

In the situation of the last lemma, we obtain a new presentation of the knot, with one 0 -handle and one 1-handle fewer. The new presentation of $\pi_{1}(K)$ is obtained by striking out $x_{i}$ and $r_{j}$, and substituting $x_{k}$ for $x_{i}$ wherever it occurs in the remaining relations.

Lemma 3.5. Suppose that two 1-handles yield the relations $y=w \times w^{-1}$, $x=u z u^{-1}$. Then we may move one of the bundles over the other to obtain the relations $y=\mathrm{uuzu}^{-1} w^{-1}, x=u z u^{-1}$.

Proof. This is an application of Lemma 2.2. 
4. Elimination of 0 -handles. A knot will be called 1-simple if $\pi_{1}(K) \cong$ ( $t$ : ).

THEOREM 4.1. If $n>3, a$ 1-simple $n$-knot has a presentation with just one 0 -handle.

Proof. Take an arbitrary presentation, with $\tau$ defined as in $\S 3$, and consider the presentation of $\pi_{1}(K)$ that this defines. If there is more than one 0 handle, there must be a relation $y=v^{-1} x v$ with $x \neq y$. Recall that $\varphi(x)=t$, and define $m$ by $\varphi(v)=t^{-m}$. Then $v x^{m}$ is null-homotopic in $K$, and so in $K_{\tau}$, by Lemma 3.1.

Adjoin a 1-handle to the 0 -handle corresponding to $x$, using the word $u x^{m}$ to describe the path of the core. Because $v x^{m}$ is null-homotopic in $K_{\tau}$, and $n>3$, this extra 1-handle may be trivially cancelled by a 2-handle. The relation yielded by this extra 1-handle is $x=v x^{m} x x^{-m} v^{-1}$, which by the results of $\S 3$ we may write as $x=v x v^{-1}$.

Applying Lemma 3.5 to the relations $y=v^{-1} x v, x=v x v^{-1}$ we obtain $y=v^{-1} v x v^{-1} v$, which by the results of $\S 3$ we may simplify to $y=x$.

Now the 0 -handle corresponding to $x$ may be cancelled, by Lemma 3.4, and iteration completes the proof.

5. Statement of the presentation theorem. An $n$-knot will be called $r$ simple if $\pi_{i}(K) \cong \pi_{i}\left(S^{1}\right)$ for $0 \leqslant i \leqslant r$.

A presentation of an $n$-knot will be called r-simple if it has just one handle of $S^{n}$ of index less than $r$ (this being a 0 -handle).

Thus Theorem 4.1 shows that for $n>3$ any 1 -simple $n$-knot has a 1 simple presentation.

THEOREM 5.1. Consider an $(r-1)$-simple presentation of an $r$-simple $n$ knot. If $2<2 r<n$, then we may ambient isotop the presentation to be $r$ simple.

The proof of this theorem will occupy the next few sections. First we examine some of the algebra involved.

From the theory of covering spaces, $\pi_{i}(K) \cong \pi_{i}(\widetilde{K}), i>1$. Thus by the Hurewicz theorem, $H_{i}(\widetilde{K}) \cong \pi_{i}(\widetilde{K})=0$ for $0<i \leqslant r$.

The knot group $\pi_{1}(K)=(t:)$ acts on $\widetilde{K}$ by covering transformations, and this action may be extended linearly to make $H_{*}(\widetilde{K})$ a $\mathrm{Z}[t]$-module in each dimension. In particular, $H_{r}(\widetilde{K})$ is the zero module over $\mathbf{Z}[t]$.

If the matrix $A$, with entries in $\mathbf{Z}[t]$, presents $H_{r}(\widetilde{K})$ as a $\mathbf{Z}[t]$-module, then $A$ is equivalent under the following moves to a matrix with no columns.

(i) Addition of a multiple of one row (column) to another row (column). 
(ii) Bordering the matrix: $A \mapsto\left(\begin{array}{ll}A & 0 \\ 0 & 1\end{array}\right)$.

(iii) The inverse of (ii).

(iv) Addition of a row of zeros to the matrix.

(v) Permutation of rows (columns).

The reader is referred to Zassenhaus [13, pp. 117-120] for details; see also Fox [2].

We shall obtain such a presentation matrix, with each column corresponding to an $(r-1)$-handle of the knot and each row to an $r$-handle. Further, we shall show that the matrix $A$ determines the positions of the $(r-1)$ and $r$-handles up to isotopy in their levels, and that the matrix moves above may be realized by embedded handle moves.

Before we embark on the geometry, note that in (i) it is sufficient that the multiple referred to should be of the form $\pm t^{m}$.

6. A geometric construction. Throughout this section we shall assume $2<2 r \leqslant n+1$. Consider a handle decomposition of $S^{n}$ with one 0 -handle $B^{n}, k(r-1)$-handles $h_{i}^{r-1}(1 \leqslant i \leqslant k), k r$-handles $h_{i}^{r}(1 \leqslant i \leqslant k)$, and one $n$-handle. Suppose that $h_{i}^{r}$ cancels $h_{i}^{r-1}$, and that the set of $n$-balls $h_{i}^{r-1} \cup$ $h_{i}^{r}(1 \leqslant i \leqslant k)$ are mutually disjoint.

Let $P=B^{n} \cup U_{1}^{k} h_{i}^{r-1}$, and $M=\partial P$. Then $M$ has a regular neighborhood in $S^{n}$ of the form $B^{1} \times M$, and from the handle decomposition described above we obtain a handle decomposition of $S^{n}$ on $B^{1} \times M$ with $k r$-handles, $k(n-r+1)$-handles, and two $n$-handles.

Embed $S^{n}$ in $S^{n+1}$ as the equatorial $n$-sphere. Then $S^{n}$ has a regular neighborhood of the form $B^{1} \times S^{n}$, and so $M$ has a regular neighborhood $N$ in $S^{n+1}$ of the form $B^{1} \times B^{1} \times M=B^{2} \times M$. Moreover, we obtain a handle decomposition of $B^{1} \times S^{n}$ on $B^{2} \times M$ with $k r$-handles, $k(n-r+1)$ handles, and two $n$-handles: each handle is of the form $B^{1} \times$ (handle of $S^{n}$ on $\left.B^{1} \times M\right)$.

If $Q=\operatorname{cl}\left[S^{n+1}-B^{2} \times M\right]$, then by adding two $(n+1)$-handles to $B^{1} \times S^{n}$ we obtain a handle decomposition of $Q$ on $B^{2} \times M$.

In this situation we shall say that $P$ and $M$ are unknotted in $S^{n+1}$.

The circle factor in $\partial Q=\partial N=S^{1} \times M$ gives a subgroup $\pi_{1}\left(S^{1}\right)$ of $\pi_{1}(\partial Q)=\pi_{1}\left(S^{1}\right) \oplus \pi_{1}(M)$ and of $\pi_{1}(Q)$. Let $\sim$ denote the covering corresponding to this subgroup; then $\partial \widetilde{Q}=\widetilde{\partial Q}$. If $\pi_{1}\left(S^{1}\right)=(t:)$, then $H_{*}(\widetilde{Q})$ and $H_{*}(\widetilde{Q}, \partial \widetilde{Q})$ are $\mathrm{Z}[t]$-modules in each dimension. In the sequel we shall assume $S^{n+1}$ and $S^{n}$ to be oriented, and that $t$ is chosen to correspond with the negative normal of $P$ in $S^{n+1}$.

Lemma 6.1. $(Q, \partial Q)$ is $(r-1)$-connected. 
LEMMA 6.2. If $2 r<n+1$, then $H_{r}(\widetilde{Q}, \partial \widetilde{Q})=\bigoplus_{1}^{k} \mathrm{Z}[t]$, with basis $\left[x_{i}\right]_{1}^{k}$ given by the cores of the r-handles, and $H_{n-r+1}(\widetilde{Q}, \partial \widetilde{Q})=\bigoplus_{1}^{k} Z[t]$ with basis $\left[y_{i}\right]_{1}^{k}$ given by the cores of the $(n-r+1)$-handles.

If $2 r=n+1$, then $H_{r}(\widetilde{Q}, \partial \widetilde{Q})=\bigoplus_{1}^{2 k} Z[t]$, with basis $\left[x_{i}\right]_{1}^{k} \cup\left[y_{i}\right]_{1}^{k}$ given by the cores of the $r$ and $(n-r+1)$-handles.

Proofs. In each case by the handle decomposition.

LEMMA 6.3. The following sequence is exact:

$$
0 \rightarrow H_{r}(\widetilde{Q}) \stackrel{i_{*}}{\longrightarrow} H_{r}(\widetilde{Q}, \partial \widetilde{Q}) \stackrel{\partial_{*}}{\longrightarrow} H_{r-1}(\partial \widetilde{Q}) \rightarrow 0 .
$$

Proof. By the long exact sequence of $(\widetilde{Q}, \partial \widetilde{Q})$,

$$
H_{r}(\partial \widetilde{Q}) \stackrel{\beta_{*}}{\longrightarrow} H_{r}(\widetilde{Q}) \stackrel{i_{*}}{\longrightarrow} H_{r}(\widetilde{Q}, \partial \widetilde{Q}) \stackrel{\partial_{*}}{\longrightarrow} H_{r-1}(\partial \widetilde{Q}) \stackrel{\beta_{*}}{\longrightarrow} H_{r-1}(\widetilde{Q})
$$

is exact. $\operatorname{Im} \beta_{*}=0$, for $H_{*}(\partial \widetilde{Q})$ is generated by the boundaries of the attaching spheres of handles of $(Q, \partial Q)$.

Now we must digress a little in order to define an intersection $S$ on $\widetilde{Q}$ regarded as a manifold with operators. Let $T$ denote the ordinary intersection of chains in a manifold; in the case of $\widetilde{Q}$ we may regard $T$ as an intersection on the homology classes as $H_{*}(\widetilde{Q}, \partial \widetilde{Q})$ is a free $\mathbf{Z}[t]$-module and so, by the handle decomposition, is $H_{*}(\widetilde{Q})$.

If $x \in H_{s+1}(\widetilde{Q}, \partial \widetilde{Q})$ and $v \in H_{n-s}(\widetilde{Q})$, define

$$
S(x, v)=\sum_{-\infty<k<\infty} T\left(x, t^{k} v\right) t^{k} \in \mathrm{Z}[t] .
$$

Define $\bar{t}=t^{-1}$, and extend this to an automorphism of $\mathrm{Z}[t]$ by linearity. Then $S$ enjoys the following properties.

1. $S(x+y, v)=S(x, v)+S(y, v)$,

2. $S(x, w+v)=S(x, w)+S(x, v)$,

3. $S(\alpha x, \bar{\beta} v)=\alpha \beta S(x, v)$,

4. $S\left(i_{*} u, v\right)=(-1)^{(s+1)(n-s)} \overline{S\left(i_{*} v, u\right)}$, where $x, y \in H_{s+1}(\widetilde{Q}, \partial \widetilde{Q}), v, w \in H_{n-s}(\widetilde{Q}), u \in H_{s+1}(\widetilde{Q}), \alpha, \beta \in \mathbf{Z}[t]$, and $i_{*}: H_{*}(\widetilde{Q}) \rightarrow H_{*}(\widetilde{Q}, \partial \widetilde{Q})$.

This intersection is used by Blanchfield [1].

LEMMA 6.4. (i) If $2 r<n+1$, then $H_{r}(\widetilde{Q})=\bigoplus_{1}^{k} \mathrm{Z}[t]$, with a basis $\left[u_{i}\right]_{1}^{k}$ such that, for $1 \leqslant i, j \leqslant k$,

$$
i_{*} u_{i}=(1-t) x_{i}, \quad S\left(y_{i}, u_{j}\right)=\delta_{i j} .
$$

(ii) If $2 r=n+1=2 q$, then $H_{q}(\widetilde{Q})=\bigoplus_{1}^{2 k} \mathrm{Z}[t]$, with a basis $\left[u_{i}\right]^{k} \cup$ $\left[v_{i}\right]_{1}^{k}$ which has the following properties for $1 \leqslant i, j \leqslant k$ : 


$$
\begin{aligned}
i_{*} u_{i} & =(1-t) x_{i}, & i_{*} v_{i} & =\left(1-t^{-1}\right) y_{i}, \\
S\left(x_{i}, u_{j}\right) & =0, & S\left(y_{i}, u_{j}\right) & =(-1)^{q} \delta_{i j}, \\
S\left(x_{i}, v_{j}\right) & =\delta_{i j}, & S\left(y_{i}, v_{j}\right) & =0 .
\end{aligned}
$$

Proof. (i) We shall examine the case $k=1$; the general case is similar. Recall the original handle decomposition of $S^{n}$ on $B^{n}$, and let $B^{r} \times B^{n-r}$ be the $r$-handle. Note that the generator $t$ of $\pi_{1}(Q)$ corresponds to the positive normal of $B^{r} \times B^{n-r}$, as it corresponds to the negative normal of $P$.

$B^{r} \times B^{n-r}$ has a regular neighborhood in $S^{n+1}, \bmod B^{r} \times \partial B^{n-r}$, of the form $B^{1} \times(1+\epsilon) B^{r} \times B^{n-r}$ where $\epsilon$ is small and positive. $B^{1}$ is oriented as the positiye normal. By choosing $N\left(=N\left(M ; S^{n+1}\right)\right)$ and $\epsilon$ carefully, we may arrange for

$$
N \cap\left[B^{1} \times(1+\epsilon) B^{r} \times B^{n-r}\right]=B^{1} \times \operatorname{cl}\left[(1+\epsilon) B^{r}-(1-\epsilon) B^{r}\right] \times B^{n-r} .
$$

We have already chosen $x_{1}$, i.e., we have chosen an orientation and lift of the ball $Q \cap\left(B^{r+1} \times 0\right)$. Now we claim that $\partial\left[B^{1} \times(1+\epsilon) B^{r} \times 0\right]$ represents $(1-t) x_{1}$ in $H_{r}(\widetilde{Q}, \partial \widetilde{Q})$. For $1 \times(1+\epsilon) B^{r} \times 0$ contributes $x_{1}$, and $-1 \times(1+\epsilon) B^{r} \times 0$ contributes $-t x_{1}$, because to get from one part to the other it is necessary to go once around the $S^{1}$ factor of $\partial Q=S^{1} \times M$ in the positive direction. The part $B^{1} \times \partial(1+\epsilon) B^{r} \times 0$ lies in $\partial Q$, and so contributes nothing. Thus if we take $u_{1}$ to be represented by $\partial\left[B^{1} \times(1+\epsilon) B^{r} \times 0\right]$, we have $i_{*} u_{1}=(1-t) x_{1}$.

From the handlebody structure of $Q$, it follows that $H_{r}(\widetilde{Q})$ is a free $\mathrm{Z}[t]$-module with basis element $u_{1}$.

Recall the handle decomposition of $S^{n}$ on $B^{n}$, and let $B^{r-1} \times B^{n-r+1}$ be the $(r-1)$-handle. $Q \cap\left(0 \times B^{n-r+1}\right)$ is the core of the $(n-r+1)$-handle in the handle decomposition of $Q$ on $B^{2} \times M$, and so $y_{1}$ is represented by an orientation and lift of $Q \cap\left(0 \times B^{n-r+1}\right)$. Because $h_{1}^{r}$ cancels $h_{1}^{r-1}$, $B^{r} \times 0$ and $0 \times B^{n-r+1}$ intersect in a single point on their boundaries, in $M$. Therefore $B^{1} \times \partial(1+\epsilon) B^{r} \times 0$ meets $0 \times B^{n-r+1}$ in a single point, in $\partial Q$, and so $\partial\left[B^{1} \times(1+\epsilon) B^{r} \times 0\right]$ meets $Q \cap\left(0 \times B^{n-r+1}\right)$ in a single point. By choosing a suitable orientation and lift of $Q \cap\left(0 \times B^{n-r+1}\right)$, we obtain $S\left(y_{1}, u_{1}\right)=1$.

(ii) We use the same argument to construct $u_{1}$ with $i_{*} u_{1}=(1-t) x_{1}$. A similar argument enables us to construct $v_{1}$ with $i_{*} v_{1}=\left(1-t^{-1}\right) y_{1}$, noting that $t^{-1}$ occurs instead of $t$ because $t$ corresponds to the negative normal of $P$. Let $B^{q}$ be the core corresponding to $x_{1}, B^{q}$ that corresponding to $y_{1}$ : thus $x_{1}$ is determined by an orientation and lift of $B^{q} \cap Q, y_{1}$ by an orientation and lift of $\underline{B}^{q} \cap Q$. Orient $\underline{B}^{q}$ so that $T\left(\partial B^{q}, \partial \underline{B}^{q}\right)=-1$, this intersection being in $M$. 
Now in the construction of $v_{1}$, we choose $N$ and $\epsilon$ so that $B^{1} \times$ $\partial(1+\epsilon) \underline{B}^{q}$ lies in $\partial Q$. Thus the sphere $\partial\left[B^{1} \times(1+\epsilon) \underline{B}^{q}\right]$ representing $v_{1}$ meets $B^{q} \cap Q$ in a single point. We need a change of sign because $\left(1-t^{-1}\right)$ occurs in the construction of $v_{1}$, instead of $(1-t)$, and so for a suitable lift $y_{1}$ of $\underline{B}^{q} \cap Q$ we have $S\left(x_{1}, v_{1}\right)=1$.

From the constructions, it is clear that $S\left(x_{1}, u_{1}\right)=0$ and $S\left(y_{1}, v_{1}\right)=0$, and it only remains to calculate $S\left(y_{1}, u_{1}\right)$.

$$
\begin{aligned}
\left(1-t^{-1}\right) S\left(y_{1}, u_{1}\right) & =S\left(\left(1-t^{-1}\right) y_{1}, u_{1}\right)=S\left(i_{*} v_{1}, u_{1}\right) \\
& \left.=(-1)^{q} \overline{S\left(i_{*} u_{1}, v_{1}\right.}\right)=(-1)^{q} \overline{S\left((1-t) x_{1}, v_{1}\right)} \\
& =(-1)^{q}\left(1-t^{-1}\right) .
\end{aligned}
$$

Therefore $S\left(y_{1}, u_{1}\right)=(-1)^{q}$.

LEMMA 6.5. If $2 r=n+1=2 q$, then $H_{q-1}(\partial \widetilde{Q})=\bigoplus_{1}^{2 k} \mathrm{Z}$, with a basis $\left[\partial_{*} x_{i}\right]_{1}^{k} \cup\left[\partial_{*} y_{i}\right]_{1}^{k}$ given by the boundaries of the cores of the $r$ and $(n-r+1)$-handles.

There is an isomorphism $H_{q-1}(\partial \widetilde{Q}) \rightarrow H_{q-1}(M)$, and if $\partial x_{i}, \partial y_{i}$ are the images under this map of $\partial_{*} x_{i}, \partial_{*} y_{i}$, then the bilinear form

$$
T: H_{q-1}(M) \times H_{q-1}(M) \rightarrow \mathrm{Z}
$$

defined by intersections is given by the formula $T\left(\partial x_{i}, \partial y_{i}\right)=-\delta_{i j}$.

Proof. The first part follows from the handle structure. As $\partial Q=S^{1} \times$ $M$, we have $\partial \widetilde{Q}=R^{1} \times M$; thus there is an obvious isomorphism $H_{q-1}(\partial \widetilde{Q})$ $\rightarrow H_{q-1}(M)$. The proof of Lemma 6.4(ii) shows that $T$ has the desired form.

7. Handle lemmas.

Lemma 7.1. Let $\left(B^{r} \times B^{n-\eta}\right)_{i}$ be proper submanifolds of $B^{n+1}$, for $i=1,2$. Assume that $\left(\partial B^{r} \times B^{n-r}\right)_{1}=\left(\partial B^{r} \times B^{n-\eta}\right)_{2}$ and that $\left(B^{n+1}\right.$, $\left.\left(B^{r} \times B^{n-\eta}\right)_{i}\right)$ is isomorphic to the standard ball pair for each $i$. If $0<2 r<n$ and $n-r \geqslant 3$, then $\left(B^{r} \times B^{n-r}\right)_{1}$ is ambient isotopic to $\left(B^{r} \times B^{n-\eta}\right)_{2}$ keeping $\left(\partial B^{r} \times B^{n-\eta} \eta_{1}\right.$ fixed.

Proof. Let $h_{i}:\left(B^{n+1},\left(B^{r} \times B^{n-r}\right)_{i}\right) \rightarrow\left(B^{n} \times B^{1}, B^{n} \times 0\right)$ be an isomorphism of the given ball pair to the standard ball pair, taking $\left(B^{r} \times B^{n-r}\right)_{i}$ productwise onto $B^{r} \times B^{n-r} \times 0=B^{n} \times 0$, for $i=1,2$. We may assume that $h_{1}^{-1} h_{2}$ is orientation preserving.

Let $C_{i}$ denote $h_{i}^{-1}\left[\left(\partial B^{n} \times[0,1]\right) \cup\left(B^{n} \times 1\right)\right]$, and let $D_{i}$ denote the closed complement in $\partial B^{n+1}$ of $h_{i}^{-1}\left(\partial B^{r} \times B^{n-r} \times[-1,0]\right)$.

As $h_{i}^{-1}\left(B^{r} \times B^{n-r} \times B^{1}\right)$ is a regular neighborhood in $\partial B^{n+1}$ of $\left(\partial B^{r} \times B^{n-r}\right)_{i} \bmod \left(\partial B^{r} \times \partial B^{n-\eta}\right)_{i}$, and $h_{1}^{-1} h_{2}$ is orientation preserving, we 
may by regular neighborhood theory ambient isotop $D_{1}$ to coincide with $D_{2}$, keeping $\left(\partial B^{r} \times B^{n-\eta}\right)_{1}$ fixed. Extend this to an ambient isotopy of $B^{n+1}$ by coning.

Consider $B_{i}^{r}=h_{i}^{-1}\left[\left(\partial B^{r} \times 0 \times[0,1]\right) \cup\left(B^{r} \times 0 \times 1\right)\right] . B_{i}^{r}$ is a proper submanifold of $C_{i}$ and of $D_{i}$. The homotopy classes of $B_{i}^{r} \subset D_{1}$, keeping $\partial B_{i}^{r}$ fixed, correspond to elements of

$$
\pi_{r}\left(S^{r} \times S^{n-r}\right)=\pi_{r}\left(S^{r}\right) \oplus \pi_{r}\left(S^{n-r}\right), \text { for } D_{1} \cong B^{r} \times S^{n-r} \text {. }
$$

The part which concerns us is $\pi_{r}\left(S^{n-r}\right)$, for we always get $\pm 1 \in \pi_{r}\left(S^{r}\right)$ according to orientation. As $2 r<n, \pi_{r}\left(S^{n-r}\right)=0$, and so $B_{1}^{r}$ is homotopic to $B_{2}^{r}$ keeping the boundary fixed. By [3, Theorem 10.1], $B_{1}^{r}$ is isotopic to $B_{2}^{r}$ keeping the boundary fixed. As $n-r \geqslant 3$, this may be covered by an ambient isotopy. Now $C_{i}$ is a regular neighborhood of $\left.B_{i}^{r} \cup\left(\partial B^{r} \times B^{n-\eta}\right)_{i}\right) \bmod$ $\left(\partial B^{r} \times \partial B^{n-\eta}\right)_{i}$ in $\partial B^{n+1}$, and so by the uniqueness of relative regular neighborhoods we may ambient isotop $C_{1}$ to $C_{2}$ keeping $\left(\partial B^{r} \times B^{n-\eta}\right)_{1}$ fixed. Again, these extend to ambient isotopies of $B^{n+1}$ by coning.

Define $E_{i}$ to be $h_{i}^{-1}\left(B^{n} \times[0,1]\right): E_{i}$ is a regular neighborhood of $C_{i}$ $\bmod \partial C_{i}$ in $B^{n+1}$, and so $E_{1}$ may be ambient isotoped to $E_{2}$ keeping $C_{1}$ fixed. As $\left(B^{r} \times B^{n-r}\right)_{i}=h_{i}^{-1}\left(B^{n} \times 0\right)$, this completes the proof.

Consider and $(r-1)$-simple presentation of an $n$-knot, so that $M$ is unknotted in $S^{n+1}$.

LEMMA 7.2. Suppose that the cores of two r-handles in normal position coincide. If $0<2 r<n, n-r \geqslant 3$, then we may ambient isotop one r-handle onto the other.

Proof. By the theory of regular neighborhoods, there is an ambient isotopy of $M$ which throws the attaching tube of one handle onto that of the other handle, keeping the attaching sphere fixed. As the handles are in normal position, this extends by the method of Lemma 2.2 to an ambient isotopy of $S^{n+2}$ keeping the core fixed.

Let $Q$ be defined as in $\S 6$, and let $\left(B^{r} \times B^{n-r}\right)_{i}$ be the intersection of the $i$ th $r$-handle with $Q$, for $i=1,2$. Let $N_{i}$ be a regular neighborhood of $\left(B^{r} \times B^{n-\eta}\right)_{i}$ mod $\left(B^{r} \times \partial B^{n-r}\right)_{i}$ in $Q$ which meets the boundary regularly. As the two attaching tubes coincide, we may assume that $N_{1} \cap \partial Q=N_{2} \cap \partial Q$. Now $N_{i}$ is also a regular neighborhood of $\left(B^{r} \times 0\right)_{i} \cup\left(\partial B^{r} \times B^{n-\eta}\right)_{i} \bmod$ $\left(\partial B^{r} \times \partial B^{n-\eta}\right)_{i}$ which meets the boundary regularly. As the two cores and the two attaching tubes coincide, we may ambient isotop $N_{1}$ onto $N_{2}$ keeping $\partial Q$ fixed. Now we may apply Lemma 7.1 to isotop one handle onto the other, keeping $\left(\partial B^{r} \times B^{n-r}\right)_{1}$ fixed. Extend the ambient isotopy to $S^{n+1}$ keeping $M$ fixed. 
LEMmA 7.3. Let $Q$ be defined as in $\S 6$, the closed complement of a regular neighborhood of $M$ unknotted in $S^{n+1}$. Suppose that $f, g: B^{r} \rightarrow Q$ are two proper embeddings representing the same element of $\pi_{r}(Q, \partial Q)$, and that

$$
f\left(S^{r-1}\right), g\left(S^{r-1}\right) \subset M_{1}=\text { point } \times M \subset S^{1} \times M=\partial Q \text {. }
$$

Then, if $2<2 r<n, f$ is ambient isotopic through embeddings $\left(B^{r}, S^{r-1}\right)$ $\rightarrow\left(Q, M_{1}\right)$ to $\bar{f}$, where $\bar{f} \simeq g$ rel $S^{r-1}$.

Proof. From the exact sequence of $\left(\partial Q, M_{1}\right) \cong\left(S^{1} \times M, M\right)$ we obtain $\pi_{i}\left(\partial Q, M_{1}\right)=0$, for $i>1$. Then the exact sequence of $\left(M_{1}, \partial Q, Q\right)$ gives

$$
\pi_{r}\left(\partial Q, M_{1}\right) \rightarrow \pi_{r}\left(Q, M_{1}\right) \rightarrow \pi_{r}(Q, \partial Q) \rightarrow \pi_{r-1}\left(\partial Q, M_{1}\right)
$$

and so $\pi_{r}\left(Q, M_{1}\right) \rightarrow \pi_{r}(Q, \partial Q)$ is an injection. Thus $f$ and $g$ represent the same element of $\pi_{r}\left(Q, M_{1}\right)$. Let $F$ be a homotopy between them,

$$
F:\left(B^{r}, S^{r-1}\right) \times I \rightarrow\left(Q, M_{1}\right) \times I .
$$

Consider $\left.F\right|_{S^{r-1} \times I}: S^{r-1} \times I \rightarrow M_{1} \times I$. By general position, as $2 r-$ $n<0$, this may be homotoped to an embedding keeping $\left.F\right|_{S r-1 \times \partial I}$ fixed. The codimension is $n-r \geqslant r+1 \geqslant 3$, and as concordance implies isotopy is codi-.. mension 3, we may homotop $\left.F\right|_{S^{r-1} \times I}$ to be an isotopy, keeping $\left.F\right|_{S^{r-1} \times \partial I}$ fixed.

Using collars of $S^{r-1}$ in $B^{r}$ and $\partial Q$ in $Q$, with the observation above, we see that $f$ is isotopic to the required $\bar{f}$. As we are in codimension 3 , this isotopy may be covered by an ambient isotopy.

8. Presentation of homology. Let $\sigma$ be a level between the $(r-1)$ and $r$-handles of $S^{n}$ in a presentation of an $n$-knot, and $\tau$ a level between the $r$ and $(r+1)$-handles. The following result is proved in the same way as Lemma 4.1.

LEMMA 8.1. The map induced by inclusion, $i_{*}: H_{r}\left(\widetilde{K}_{\tau}\right) \rightarrow H_{r}(\widetilde{K})$, is an isomorphism if $2 r<n$.

Suppose that the presentation is $(r-1)$-simple, and that after the $k(r-1)$ handles have been added we are left with a manifold $M$ unknotted in $S^{n+1}$ as in §6. Let $Q$ be the closed complement in $S^{n+1}$ of a regular neighborhood $N$ of $M$; thus $Q \cong K_{\sigma}$. Assume that $M$ is in a level above $\sigma$, and that we slide the $m r$-handles of $S^{n}$ down into this level.

Let $\left(B^{r} \times B^{n-\eta}\right)_{i}$ denote the $i$ th $r$-handle: by $\S 2$ we may arrange for them to be in normal position; in particular we may assume that $Q \cap\left(B^{r} \times 0\right)_{i}$ is a ball for each $i$. Choosing an orientation for $\left(B^{r} \times 0\right)_{i}$, and a representative lift in $\widetilde{Q}$ of $Q \cap\left(B^{r} \times 0\right)_{i}$, we obtain an element of $H_{r}(\widetilde{Q}, \partial \widetilde{Q})$. 
LEMMA 8.2. Suppose that $2<2 r<n$, and that $H_{r}(\widetilde{Q}), H_{r}(\widetilde{Q}, \partial \widetilde{Q})$ are based as in §6. If $Q \cap\left(B^{r} \times 0\right)_{i}$ represents $\alpha_{i j} x_{j} \in H_{r}(\widetilde{Q}, \partial \widetilde{Q})$, then

$$
H_{r}\left(\widetilde{K}_{\tau}\right)=\left[u_{1}, \cdots, u_{k}: \alpha_{i j} u_{j}=0,1 \leqslant i \leqslant m\right]
$$

as a $\mathrm{Z}[t]$-module.

Proof. Being in normal position, the $i$ th $r$-handle has a regular neighborhood of the form $B^{1} \times(1+\epsilon)\left(B^{r} \times B^{n-\eta}\right)_{i}$, where $\epsilon$ is small and positive, and which meets $N$ in

$$
B^{1} \times\left(\mathrm{cl}\left[(1+\epsilon) B^{r}-(1-\epsilon) B^{r}\right] \times(1+\epsilon) B^{n-\eta}\right)_{i} .
$$

Let $X$ be the closure of the space obtained from $S^{n+1}$ by excising both $N$ and the regular neighborhoods of the $r$-handles described above. We have the following homotopy equivalences, proved by deformation retracting the balls $B^{1} \times(1+\epsilon)\left(B^{r} \times B^{n-r}\right)_{i}$ onto appropriate subspaces:

$$
\begin{aligned}
& Q \simeq X \cup \bigcup_{1}^{m} B^{1} \times(1+\epsilon)\left(0 \times B^{n-\eta}\right)_{i}, \\
& K_{\tau} \simeq X \cup \bigcup_{1}^{m} B^{1} \times(1+\epsilon)\left(B^{r} \times 0\right)_{i} .
\end{aligned}
$$

Therefore up to homotopy equivalence, $Q$ is obtained from $X$ by adding some $(n-r+1)$-cells: as $2 r<n$ implies $n-r+1>r+1, H_{r}(\widetilde{X}) \cong H_{r}(\widetilde{Q})=$ $\left[u_{1}, \cdots, u_{k}:\right]$.

To compute $H_{r}\left(\widetilde{K}_{\tau}\right)$, we argue just as in the proof of Lemma 6.4, obtaining the desired presentation from the second homotopy equivalence above.

REMARK. If we consider the projection from the level of the $r$-handles to the level of the $(r-1)$-handles, we may assume by transversality that the shadows $C_{i}$ of the cores of the $r$-handles meet the cocores $D_{j}$ of the $(r-1)$-handles in a finite set of points. By the argument in the proof of Lemma 6.4, we may take the matrix $A=\left(\alpha_{i j}\right)$ above to be this intersection matrix:

$$
-T\left(\partial C_{i}, \partial D_{j}\right)+(1-t) S\left(\text { int } C_{i} \text {, int } D_{j}\right)=S\left(C_{i}, \partial\left(B^{1} \times(1+\epsilon) D_{j}\right)\right) \text {. }
$$

REMARK. Let $M_{1}=* \times M \subset \partial B^{2} \times M=\partial N$, where $*$ is a point of $\partial B^{2}$. As the handles are in normal position, we may make a canonical choice of the representative lift of $Q \cap\left(B^{r} \times 0\right)_{i}$ by requiring that the boundary of this representative shall lie in some fixed lift $\widetilde{M}_{1}$ of $M_{1}$, for each $i$.

9. Proof of the presentation theorem. Recall that we have an $(r-1)$ simple presentation of an $r$-simple $n$-knot. We shall show that if there are $k$ $(r-1)$-handles and $m r$-handles of $S^{n}$, then these determine a matrix $A$ over 
$\mathrm{Z}[t]$ with $k$ columns and $m$ rows. Each column will correspond to an $(r-1)$ handle, each row to an $r$-handle. Further, we shall show that the matrix $A$ determines the positions of the $r$-handles up to ambient isotopy in their level. We assume inductively that the $(r-1)$-handles are unknotted in their level, in the sense that we may move them down to the level of the 0 -handle, to obtain a manifold $P$ unknotted in the sense of $\S 6$. Note that this is a special case of what we are trying to prove, but one dimension lower, for when there are no $(r-1)$ handles of $S^{n}$, the matrix $A$ has no columns, and so we shall be proving that the $r$-handles are unknotted in their level.

Granted this inductive hypothesis, we see that the $r$-handles are attached to a submanifold $M$ of $S^{n+1}$, where $M \cong \partial P$ is unknotted in the sense of $\S 6$. If $N \cong B^{2} \times M$ is a regular neighborhood of $M_{1}$ and $Q=\operatorname{cl}\left[S^{n+1}-N\right]$, then we use $P$ to obtain a handle decomposition of $Q$, as in $\S 6$, and hence to define bases for homology as in that section.

By $\S 8$ we obtain a presentation for $H_{r}(\widetilde{K}) . A$ is defined to be the matrix $\left(\alpha_{i j}\right)$ of Lemma 8.2.

By the Hurewicz and covering space theorems,

$$
H_{r}(\widetilde{Q}, \partial \widetilde{Q}) \cong \pi_{r}(\widetilde{Q}, \partial \widetilde{Q}) \cong \pi_{r}(Q, \partial Q) .
$$

Thus $A$, which describes the elements of $H_{r}(\widetilde{Q}, \partial \widetilde{Q})$ represented by the cores of the $r$-handles, also describes the elements of $\pi_{r}(Q, \partial Q)$ represented by the cores of these handles.

Suppose that we have two sets of $r$-handles, $h_{i}$ and $h_{i}^{\prime}, 1 \leqslant i \leqslant m$, with cores $C_{i}$ and $C_{i}^{\prime}$. Assume that after being moved into normal position, they determine the same matrix $A$. We may ambient isotop $\partial C_{i}$ to coincide with $\partial C_{i}^{\prime}$ in $M$, for they are homologous and hence homotopic by the Hurewicz theorem and, by general position, as $2 r<n$ implies $2(r-1)-(n-1)<-1$, we may realize this homotopy by an isotopy. As $2<2 r<n$ implies $(n-1)-(r-1) \geqslant$ $r+1 \geqslant 3$, this may be covered by an ambient isotopy in $M$, and this may be extended to an ambient isotopy of the knot by Lemma 2.2 .

This means that $\partial\left(Q \cap C_{i}\right)=\partial\left(Q \cap C_{i}^{\prime}\right)$, and by the remark above $(Q \cap$ $\left.C_{i}\right)$ and $\left(Q \cap C_{i}^{\prime}\right)$ represent the same element of $\pi_{r}(Q, \partial Q)$. By Lemma 7.3, we may assume that they are homotopic rel boundary, and by general position, as $2 r<n$ implies $2 r-(n+1)<-1$, this homotopy may be realized by an isotopy keeping the boundary fixed. As we are in codimension 3 , this may be covered by an ambient isotopy of $Q$, keeping $\partial Q$ fixed.

Thus we may ambient isotop $C_{i}$ to coincide with $C_{i}^{\prime}, 1 \leqslant i \leqslant n$. By Lemma 7.2, we may isotop $h_{i}$ to coincide with $h_{i}^{\prime}$ as desired.

Now we must show that the matrix moves of $\S 5$ can be realized by handle moves. 
(ii) is easy: we add a pair of cancelling handles of index $(r-1), r$ to $S^{n}$. The effect of this on $H_{r}(\widetilde{Q}, \partial \widetilde{Q})$ is to add a direct summand $\mathbf{Z}[t]$, and the effect on $A$ is to border the matrix.

(iii) is also easy, for after an isotopy we may assume that we have a configuration such as we have just described, so that by cancelling the pair of handles we effect the matrix move.

(iv) consists of the same move as (ii), except that we introduce a cancelling pair of $r,(r+1)$-handles.

Now for (i). Consider two $r$-handles $h_{i}, h_{j}$ of $S^{n}$. Move $h_{j}$ down to a level between the $(r-1)$ and $r$-handles, and consider $h_{i}$ to be the first $r$-handle added after $h_{j}$. Let $M^{\prime}$ be the boundary of the manifold to which $h_{i}$ is added. The proof of the handle addition theorem [3, Theorem 3.1] shows that we may move $h_{i}$ over $h_{j}$ by considering a ball $B^{n-1} \subset M^{\prime}$ which meets the attaching sphere of $h_{i}$ in a ball $B^{r-1}$. $\left(B^{n-1}, B^{r-1}\right)$ forms an unknotted ball pair, and there is an ambient isotopy of $B^{n-1}$, fixed on the boundary, which moves $B^{r-1}$ over $h_{j}$. Before applying this isotopy, consider a regular neighborhood $B^{2} \times T$ of $T$ mod $\partial T$, where $T$ is the attaching tube of $h_{i}$ : we assume $T$ to be disjoint from $h_{j}$. Let $g_{t}: B^{2} \rightarrow B^{2}$ be an isotopy which moves $\partial B^{2} p$ times around itself and keeps the origin fixed. Apply $g_{t} \times$ id to $B^{2} \times T$, and extend to the rest of $S^{n+1}$ by taking collars. If the cores of $h_{i}, h_{j}$ initially represented $\xi_{i}, \xi_{j} \in H_{r}(\widetilde{Q}, \partial \widetilde{Q})$, they now represent $t^{-p} \xi_{i}, \xi_{j}$ : this may be seen by considering the connection between homology and the intersection of cores and cocores in the handle decomposition of $Q$ (recall the remarks at the end of $\S 8$ ).

Now apply the handle addition isotopy, using Lemma 2.2: the cores now represent $t^{-p} \xi_{i} \pm \xi_{j}, \xi_{j}$. Next apply $g_{t}^{-1} \times$ id to $B^{2} \times T$ : now the cores represent $\xi_{i} \pm t^{p} \xi_{j}$, $\xi_{j}$. Move $h_{j}$ back up to the level of the other $r$-handles.

Similarly we may move an $(r-1)$-handle over another $(r-1)$-handle to obtain column addition.

(v) is merely relabelling.

After the remarks of $\S 5$, the proof is complete.

\section{Corollary.}

COROLlaRY 10.1. Any presentation of an r-simple $n$-knot, where $n>3$, $2 r<n$, may be ambient isotoped to an r-simple presentation of which the dual presentation is also r-simple.

Proof. Theorem 4.1 enables us to get rid of all but one of the 0 -handles and, by considering the dual presentation, to eliminate all but one of the $n$ handles. Note that in the proof of the theorem only the 0,1 and 2-handles of the knot were moved. Theorem 5.1 then enables us to cancel all the other handles 
of index less than $r$, without disturbing any handles of index greater than $r+1$. Now $2 r<n$ implies $n-r>r$ which implies $n-(r+1)>r-1$, and so by taking the dual presentation we may cancel all the other handles of index greater than $n-r$, i.e., we obtain an $r$-simple presentation of which the dual presentation is also $r$-simple.

\section{REFERENCES}

1. R. C. Blanchfield, Intersection theory of manifolds with operators with applications to knot theory, Ann. of Math. (2) 65 (1957), 340-356. MR 19, 53.

2. R. H. Fox, A quick trip through knot theory, Topology of 3-Manifolds and Related Topics (Proc. The Univ. of Georgia Inst., 1961), Prentice-Hall, Englewood Cliffs, N. J., 1962, pp. 120-167. MR 25 \#3522.

3. J. F. P. Hudson, Piecewise linear topology, Benjamin, New York, 1969. MR 40 \#2094.

4. M. C. Irwin, Embeddings of polyhedral manifolds, Ann. of Math. (2) 82 (1965), 1-14. MR $32 \# 460$.

5. C. Kearton, Classification of simple knots by Blanchfield duality, Bull. Amer. Math. Soc. 79 (1973), 952-955.

6. C. Kearton and W. B. R. Lickorish, Piecewise linear critical levels and collapsing, Trans. Amer. Math. Soc. 170 (1972), 415-424. MR 46 \#9997.

7. M. A. Kervaire, Les noeuds de dimensions supérieures, Bull. Soc. Math. France 93 (1965), 225-271. MR 32 \#6479.

8. J. Levine, Unknotting spheres in codimension two, Topology 4 (1965), 9-16. MR 31 \#4045.

9. - Polynomial invariants of knots of codimension two, Ann. of Math. (2) 84 (1966), 537-554. MR $34 \# 808$.

10. - An algebraic classification of some knots of codimension two, Comment Math. Helv. 45 (1970), 185-198. MR 42 \#1133.

11. C. P. Rourke, Embedded handle theory, concordance and isotopy, Warwick University (preprint).

12. G. Torres and R. H. Fox, Dual presentations of the group of a knot, Ann. of Math. (2) 59 (1954), 211-218. MR 15, 979.

13. H. Zassenhaus, Lehrbuch der Gruppentheorie, Teubner, Leipzig, 1937; English transl., Chelsea, New York, 1949. MR 11, 77. 